\title{
Cryo Soft X-ray Microscopy for Whole Cell Imaging - Progress in the Development of a Commercial Laboratory Scale Device
}

\author{
Kenneth Fahy ${ }^{1}$, Tony McEnroe ${ }^{1}$, Dunja Skoko ${ }^{1}$, William Fyans ${ }^{1}$, Fergal O'Reilly ${ }^{2}$ and Paul Sheridan ${ }^{1}$ \\ ${ }^{1}$ SiriusXT Ltd., Ireland, ${ }^{2}$ University College Dublin, Ireland
}

SiriusXT is developing a commercial bench-top cryo soft X-ray microscope for 3D cryo-soft X-ray tomography (cryo-SXT). Cryo-SXT uses X-rays in the 'water window' that extends from the K-absorption edge of carbon to the K-edge of oxygen, that is from about $282 \mathrm{eV}(\lambda=4.4 \mathrm{~nm})$ to $533 \mathrm{eV}(\lambda=2.3 \mathrm{~nm})$. Water is transparent to these X-rays, but organic molecules are absorbing. Therefore, these X-rays can be used as the basis for microscopy of whole cells in their near-native (frozen) state, without need for any contrast enhancing agents. A 3D tomogram with resolution between $25 \mathrm{~nm}$ to $60 \mathrm{~nm}$ (full pitch) is produced by rotating the cell over a range of angles, with an image acquired at each tilt angle $[1,2,3]$. The concept is equivalent to a medical CT scan applied at the nanoscale. Similar to Hounsfield units in medical CT, cellular organelles within the cell can be discernible from each other by their respective x-ray linear absorption coefficient values. While great progress has been made over the last two decades in developing cryo-SXT as an imaging technique on synchrotron hosted microscopes [4-7], only two laboratory soft X-ray microscopy systems have been reported so far [8-10]. The SiriusXT approach is novel insofar as no commercial lab-scale soft X-ray microscope has been available up to now, which is capable of delivering the necessary image quality and throughput required by the biomedical community. Bringing cryo-SXT capabilities to the laboratories as a table-top solution will promote development of unique flexible sample handling systems for imaging of adherent and in suspension cells, thus accelerating the establishment of integrated multiscale hybrid microscopy methods that could benefit by combining cryo-SXT with light and electron microscopy techniques [11,12]. For example, cryo-SXT could be used as a fast pre-screening tool for EM imaging, where whole single cell SXT data is used to select the region of interest to be further imaged with electron microscopy or correlated light and electron microscopy.

We will present our cryo correlative workflow in detail, including results of cryo-SXT as applied to a variety of biological specimens.

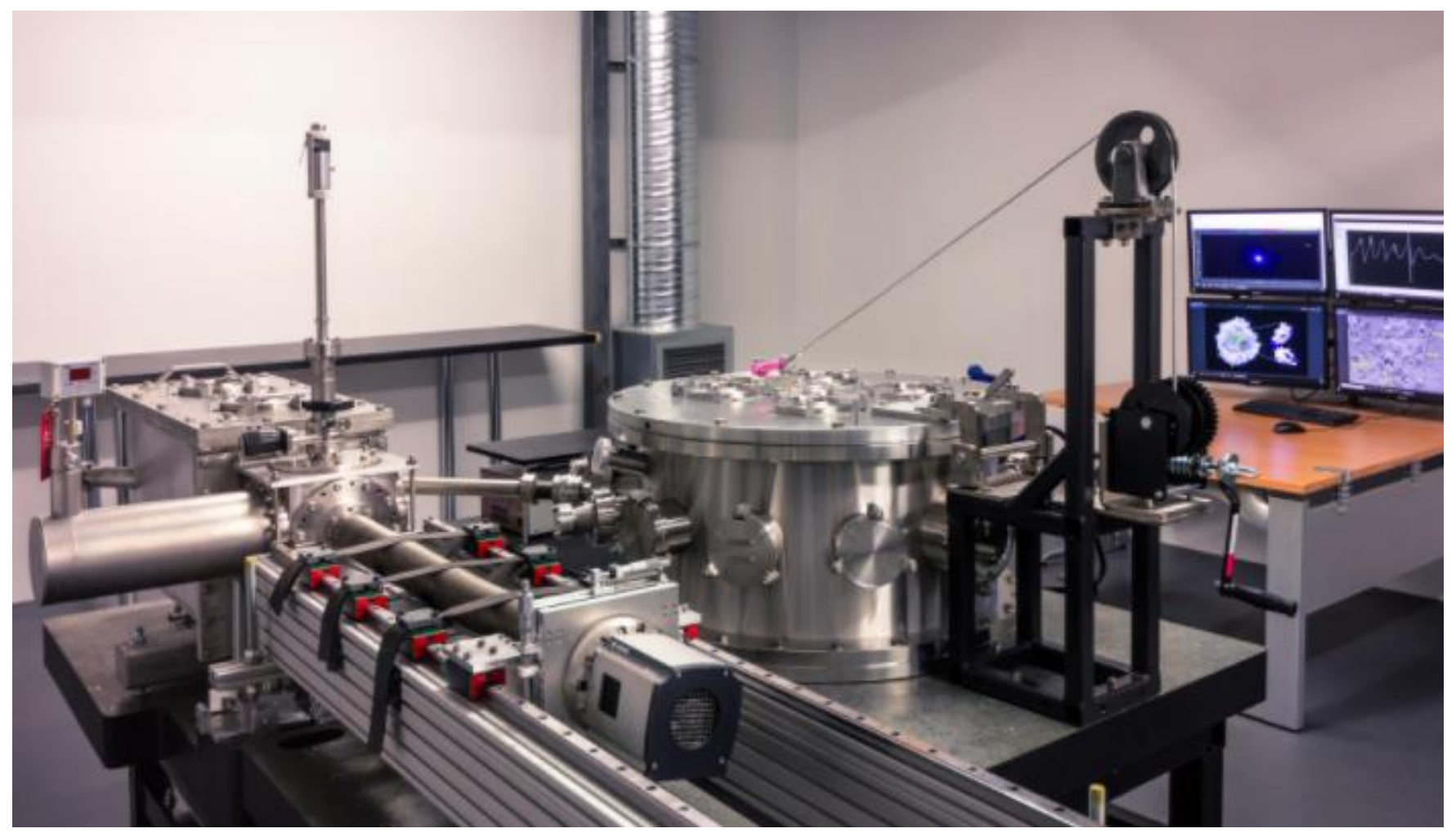


Figure 1. The laboratory soft X-ray microscope.

\section{References}

[1] Schneider G, Guttmann P, Heim S et al 2010 Nature Methods 7 985-987

[2]Müller WG, Heymann JB, Nagashima K et al 2012 J Struct Biol177 179

[3] McDermott G, Fox DM, Epperly L et al 2012 BioEssays34 320

[4] Carrascosa JL, Chichon FJ, Pereiro E et al 2009 Journal of Structural Biology168 234

[5] Larabell CA \& Nugent KA 2010 Current Opinion in Structural Biology20 623

[6] Schneider G, Guttman P, Heim S et al 2010Nature Methods 7985

[7] Carzaniga R, Domart MC, Collinson LM et al 2014 Protoplasma251 449

[8]Kördel M, Dehlinger A, Seim C et al 2020 Optica7 (6) 658-674

[9]Fogelqvist E, Kördel M, Carannante V et al 2017 Scientific Reports 7

[10] Legall H, Blobel G, Stiel H et al 2012 Optics Express 20 (16) 18362-18369

[11] Zeev-Ben-Mordehai T, Hagen C, Grunewald K 2014 Current Opinion in Virology 5 42-49

[12] Dent K, Hagen C, Grunewald K 2014 Critical Step-by-Step Approaches Toward Correlative Fluorescence/ Soft X-Ray Cryo-Microscopy of Adherent Mammalian Cells Methods in Cell Biology 124, 179-216 